\title{
Promising New Photon Detection Concepts for High-Resolution Clinical and Preclinical PET
}

\author{
Craig S. Levin \\ Molecular Imaging Instrumentation Laboratory, Departments of Radiology, Physics, and Electrical Engineering, Stanford University, \\ Stanford, California
}

The ability of PET to visualize and quantify regions of low concentration of PET tracer representing subtle cellular and molecular signatures of disease depends on relatively complex biochemical, biologic, and physiologic factors that are challenging to control, as well as on instrumentation performance parameters that are, in principle, still possible to improve on. Thus, advances to the latter can somewhat offset barriers of the former. PET system performance parameters such as spatial resolution, contrast resolution, and photon sensitivity contribute significantly to PET's ability to visualize and quantify lower concentrations of signal in the presence of background. In this report we present some technology innovations under investigation toward improving these PET system performance parameters. We focus particularly on a promising advance known as 3-dimensional position-sensitive detectors, which are detectors capable of distinguishing and measuring the position, energy, and arrival time of individual interactions of multi-interaction photon events in 3 dimensions. If successful, these new strategies enable enhancements such as the detection of fewer diseased cells in tissue or the ability to characterize lower-abundance molecular targets within cells. Translating these advanced capabilities to the clinic might allow expansion of PET's roles in disease management, perhaps to earlier stages of disease. In preclinical research, such enhancements enable more sensitive and accurate studies of disease biology in living subjects.

Key Words: PET; detector; photon sensitivity; inter-crystal scatter; 3D position; depth of interaction; high resolution; contrast resolution

J Nucl Med 2012; 53:167-170

DOI: 10.2967/jnumed.110.084343

With miniscule detection elements and high photon sensitivity, in principle PET is capable of achieving submillimeter resolution (1). Thus, during the past decade there have been considerable efforts to develop PET systems with smaller detection elements (please see 2-7 for a more extensive review of this topic). The state-of-the-art commercially available small-animal PET systems use detectors comprising arrays of $1.5-\mathrm{mm}$ width scintillation crystal elements and achieve less than 2-mm resolution at their center (2-7). The relatively high photon sensitivity of the smalldiameter animal PET system geometries help them to realize the desired high spatial resolution in reconstructed images (8).

On the other hand, the current clinical whole-body PET system geometries use a 70- to $80-\mathrm{cm}$ detector ring diameter; thus, it is futile to use smaller than 4-mm-width crystal elements in such whole-body clinical PET designs, since the photon flux per detection element is so

Received Oct. 26, 2011; revision accepted Jan. 5, 2012.

For correspondence or reprints contact: Craig S. Levin, Stanford University School of Medicine, 300 Pasteur Dr., MC 5128, Alway Building, Room M001, Stanford, CA 94305-5128.

E-mail: cslevin@stanford.edu

COPYRIGHT (c) 2012 by the Society of Nuclear Medicine, Inc.

low. The only way for clinical PET to use smaller elements and realize higher resolution in reconstructed images is to greatly improve photon sensitivity, which may be accomplished by increasing the system's geometric detection efficiency. This may be realized by bringing detectors closer to the patient, as is the case for locoregional (organ-specific) PET systems $(9,10)$, or by increasing the axial extent of the system $(4,7,11)$. In the former case, when detectors are near the subject, many photons are incident at oblique angles with respect to the detector surface. Then, the first detection element traversed by the $511-\mathrm{keV}$ photon is often not the one with which the photon first interacts, causing a misidentification of the line of response and resulting spatial resolution degradation; This is the so-called photon depth-of-interaction problem (2-7). Furthermore, since it is also highly likely that incoming $511-\mathrm{keV}$ photons undergo Compton scatter in the detector crystals, with small (e.g., $\leq 2-\mathrm{mm}$ width) detection elements, most incoming photons interact with more than one detection element $(5,12)$. But, with the standard PET detector design, such multicrystal events are not identified. This inability to accurately treat multi-interaction photon events causes uncertainty in line-ofresponse positioning that further degrades spatial resolution and also decreases resulting image contrast resolution and contrast recovery (13).

This paper focuses on describing a promising advance known as 3dimensional (3D) position-sensitive detectors, which are detectors capable of distinguishing and measuring the position, energy, and arrival time of individual interactions of multi-interaction photon events in 3 dimensions (5). With 3D positioning detectors, the photon depth of interaction is measured, enabling correct line-of-response assignment and preserved spatial resolution for obliquely entering photons that undergo only one interaction in the detector. However, there is also adequate information to accurately reconstruct the sequence of interactions for the case of a multi-interaction event. This interaction sequencing capability enables one to accurately estimate the location of the first interaction to preserve both spatial and contrast resolution in a highresolution system that uses detectors with miniscule (e.g., $<2-\mathrm{mm}$ width) detection elements (13). Furthermore, this 3D positioning of detector interactions enables new algorithms that estimate the incident direction of individual annihilation photons entering the system (14). We will describe how this photon incidence angle information, if successful, would add new and powerful capabilities for PET.

\section{CHALLENGES WITH THE CONVENTIONAL 511-KEV PHOTON DETECTOR DESIGN USED IN PET}

The conventional PET detector design (Fig. 1A) is a position-sensitive scintillation detector comprising an array of scintillation crystal elements, which are completely or partially optically isolated from each other using a reflector, with ends coupled to a position-sensitive photodetector configuration $(2-7,11,12)$. Typically to reduce the cost and complexity, the design uses scintillation light multiplexing (a.k.a. "sharing") at the bottom of the array or uses a light diffuser so that there are fewer photodetectors than crystal elements. In addition, to reduce electronic complexity, the photodetector readout circuit is typically multiplexed into just a few (e.g., 4) readout channels. There are a few challenges that arise for this standard detector design. Decreasing the crystal element width to achieve higher spatial resolution results in scin- 


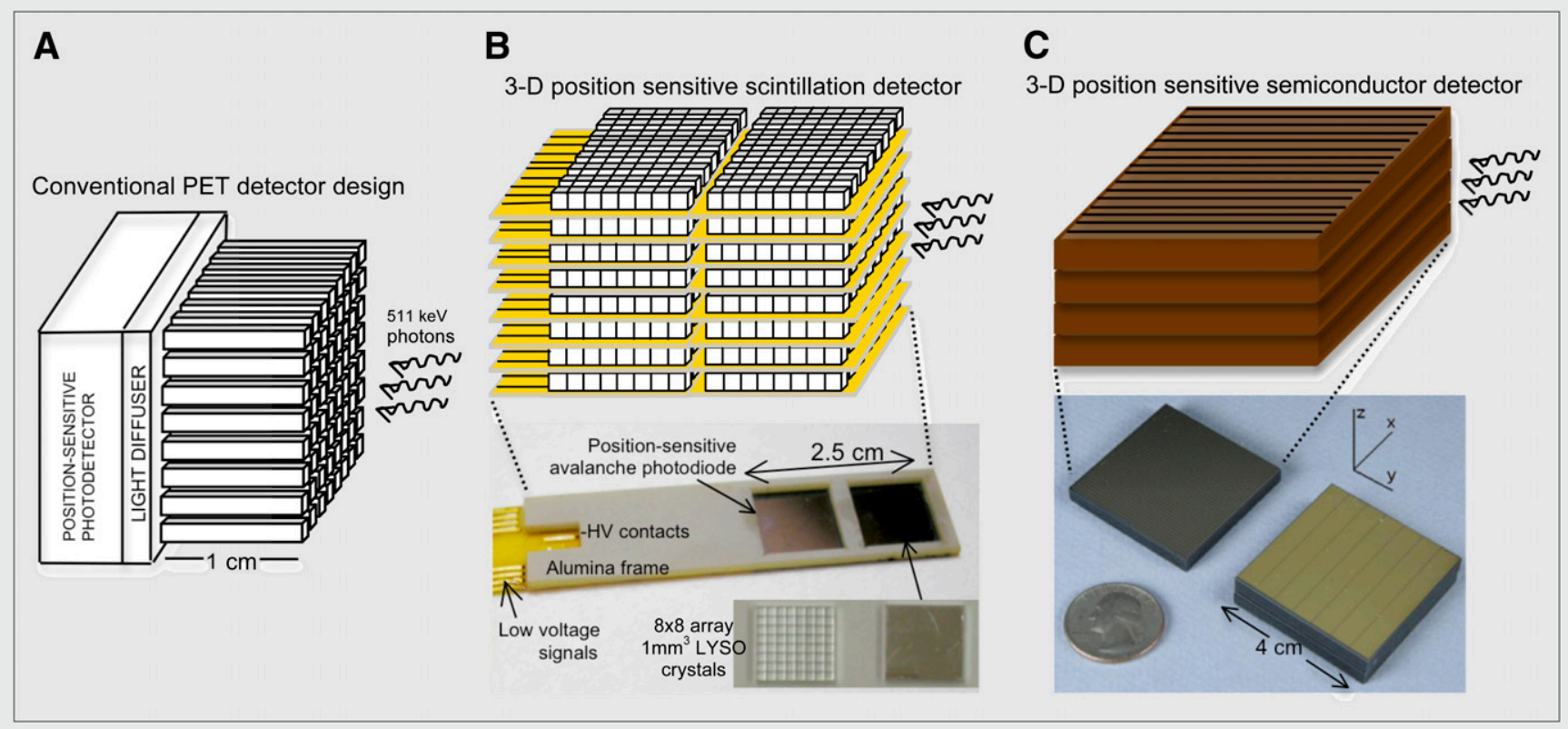

FIGURE 1. Depiction of conventional PET scintillation detector design (A), and 3D position sensitive scintillation (B) and semiconductor (C) detector concepts. In addition to measuring photon interaction depth, the latter two 3D positioning detector concepts are capable of measuring position, energy, and arrival time of each individual interaction when more than one detection element is hit. HV = high-voltage; LYSO = lutetium yttrium oxyorthosilicate.

tillation light trapping with a light collection efficiency that varies strongly with the distance between the point of light creation and the photodetector $(5,12)$. This depth-of-interaction-dependent light collection efficiency variation degrades energy resolution and time resolution performance, which are parameters that affect the ability to reject background scatter and random coincidences, thus resulting in loss of image contrast resolution, especially in patients, where those sources of background events are most significant. The resulting reduced electronic pulse height also limits the extent of electronic multiplexing that may be used.

To mitigate these light signal variation effects associated with highresolution PET, the typical approach is to reduce the length of the scintillation crystal elements to no more than $12 \mathrm{~mm}$ (Fig. 1A) $(2-7,12)$, which of course diminishes photon counts and thus signal-to-noise ratio per crystal element, limiting the ability to reconstruct images at the desired spatial resolution (8). Thus, as a result of the lower intrinsic photon detection efficiency, typically this short-crystal approach (Fig. 1A) is used in higher-photon sensitivity PET system geometries such as that for smallanimal imaging (2-4) or organ-specific clinical imagers $(9,10)$. In these system designs that use short crystal elements, the absolute photon sensitivity is only a few percent, unless the axial extent of the system is significantly increased $(4,7,11)$, with a resulting higher system cost.

A further challenge with the standard PET detector concept (Fig. 1A) is that as the crystal element width decreases, spatial resolution and contrast resolution performance are limited because of the higher fraction of photons that interact in multiple crystal elements, and the greater sensitivity to variations in photon depth of interaction. In the conventional design (Fig. 1A), because of the described light or electrical multiplexing inherent to most designs, a multicrystal photon event is positioned at a single location, at roughly the energy-weighted mean of the individual interactions, and in 2 dimensions only; and multicrystal interactions are not resolved. Many researchers have investigated detectors capable of measuring photon depth of interaction (2-7), enabling photon event positioning at a single location in 3 dimensions, rather than 2 dimensions to more accurately position oblique incidence photons for better spatial resolution uniformity throughout the field of view. However, for the standard depth-of-interaction-capable detector, multicrystal interactions are still not resolved, limiting both spatial resolution and contrast resolution performance. To date, there have been relatively few PET detector designs reported that are capable of true
$3 \mathrm{D}$ positioning of the individual interaction coordinates of annihilation photons interacting in multiple detector elements $(5,15,16)$.

\section{BEYOND THE CONVENTIONAL DESIGN: 3D POSITION-SENSITIVE 511-KEV PHOTON DETECTION}

Figures $1 \mathrm{~B}$ and $1 \mathrm{C}$ depict two 3D position-sensitive photon detectors under investigation $(15,16)$. The first (Fig. 1B) comprises a stack of several 2-dimensional scintillation detector layers comprising arrays of miniscule lutetium yttrium oxyorthosilicate crystal elements coupled to thin positionsensitive semiconductor photodetectors mounted on a flexible circuit (17). Unlike the standard design (Fig. 1A), the crystal arrays are arranged edgeon with respect to incoming $511-\mathrm{keV}$ photons to maintain high photon sensitivity. But since the dimensions of the individual crystal elements in each layer are small, the variance in scintillation light path length and transit time to the photodetector are also small, and the scintillation light collection efficiency is high $(>90 \%)$, independent of the element width, surface treatment, and interaction location (12). This minimization of optical photon path length/transit time variations and light collection efficiency variations enables one to optimize time and energy resolution performance and achieve robust scintillation detector signals. Intrinsic detector performance testing has yielded less than 1-mm intrinsic spatial resolution, depending on the detection element dimensions, approximately $10 \%$ full-width-at-half-maximum (FWHM) energy resolution at $511 \mathrm{keV}$, and from approximately 2-ns all the way down to below 300-ps FWHM coincidence time resolution, depending on the type of photodetector used $(15,18)$. Furthermore, in this scheme, submillimeter crystal elements may be used to achieve less than 1-mm spatial resolution while mitigating the usual drawbacks associated with miniscule crystals such as significant light signal loss and amplitude and temporal dispersion (12).

To realize the desired spatial resolution enhancements in reconstructed images requires increased photon sensitivity. High photon sensitivity in this new 3D position-sensitive scintillation detector design concept (Fig. 1B) is achieved by arranging the detectors edgeon with respect to incoming $511-\mathrm{keV}$ photons so that they traverse a minimum of approximately $2 \mathrm{~cm}$ of scintillation crystal material, and by configuring the system geometry into panels that can be brought close to the subject for organ-specific, locoregional clinical imaging $(9,10)$ or small-animal imaging $(19)$. This 3D positioning 


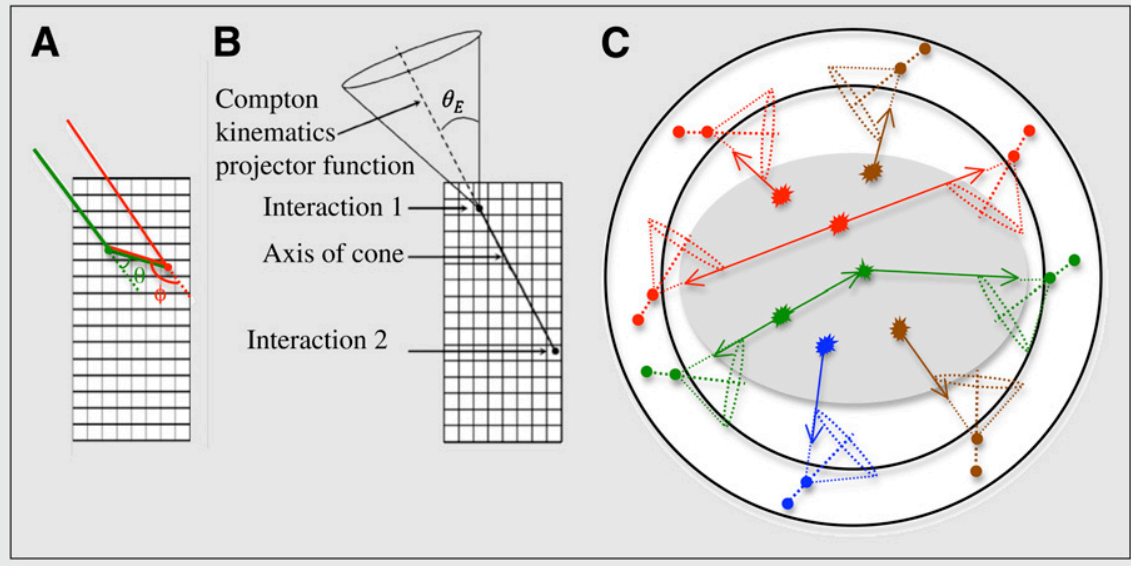

FIGURE 2. (A) Based on 3D coordinates and energy of each interaction of a multi-interaction photon event, one may estimate sequence of interactions in the detector (2 potential interaction sequences, red and green, for 2 interactions are depicted). (B) Spatial locations of 2 interactions in detector determine axis of cone formed by line of incidence of incoming photon. Using physics of Compton scatter, measured energies of interactions determine angle of cone, and therefore incoming photon direction. (C) Ability of PET detector system to accurately estimate incoming photon direction from information on individual interactions (colored dots) in detector enables new features such as extraction of true from multiple $(>2)$ photon coincidences (red) and rejection of random coincidences (brown) (14), as well as retention rather than rejection of single photons (blue) (24) or a high fraction of tissuescattered photons (green) (25).

scintillation detection concept is currently used in the design of the world's first 1-mm-resolution clinical PET system under development for dedicated locoregional cancer imaging $(15,20-22)$ as well as a new design for a submillimeter-resolution small-animal PET system.

The second approach to achieve a 3D position-sensitive PET detector uses a semiconductor crystal known as the cadmium zinc telluride (CZT) crystal (Fig. 1C) (16). A 3D positioning photon detector is achieved in CZT by metalizing the 2 large faces of each crystal slab with a segmented electrode configuration and establishing a large electric field across the device $(5,16)$. The electrode segments are each attached to isolated channels of an electronic readout system that can identify and localize current induced on any of the segments. When a $511-\mathrm{keV}$ photon interacts in the CZT, it produces ionization charge by liberating electrons from the crystal lattice, creating electron-hole pairs; the electrons will drift toward the positive electrode (anode) and the holes toward the negative electrode (cathode). The motion of the charge carriers induces a current on the closest electrode segments on either side of the crystal, enabling a method to determine the 2-dimensional coordinates of the 511-keV photon event in the plane parallel to the electrode surfaces, with the intrinsic spatial resolution along either direction defined by the pitch or spacing between electrode segments corresponding to that direction $(5,16)$. The third coordinate of the interaction, the one orthogonal to the electrode planes, is determined by a ratio of the pulse heights induced on the 2 electrode faces $(5,16)$. In the box-shaped, CZT smallanimal PET system under construction $(16,19), 4$ relatively large-area panels will be formed by stacks of these 3D positioning detectors (Fig. 1C).

Since this semiconductor crystal detector directly measures the ionization charge created by the 511-keV-photon absorption and bypasses the intermediate steps of scintillation light creation, transport, and collection into a photodetector, the electronic signal-to-noise ratio is much higher $(5,16,23)$. As a result, the CZT detector design has achieved less than 3\% FWHM energy resolution at $511 \mathrm{keV}(5,16)$. In the system design under construction (16), the electrode planes on each detector are designed as sets of parallel strips, with the anode strips at a 1-mm pitch in one direction and the cathode strips orthogonal to that at a $5-\mathrm{mm}$ pitch. With this strip configuration, the design has achieved less than 1-mm FWHM resolution in the direction orthogonal to the anode strips, less than 5-mm resolution along the direction orthogonal to cathode strips, and approximately $0.5 \mathrm{~mm}$ along the direction orthogonal to the electrode planes (16). Realizing the desired spatial resolution in reconstructed images requires increased photon sensitivity, which is achieved by arranging the detectors edge-on with respect to incoming 511-keV photons so that they traverse a minimum of approximately $4 \mathrm{~cm}$ of CZT crystal material and by bringing the 4 panels of the PET system closer to the animal (19). Simulations predict that such a configuration leads to $20 \%-30 \%$ absolute photon sensitivity at the system center, depending on an adjustable system aperture (19).

A general challenge with CZT detector designs is the relatively poor time resolution $(5,16)$, which ordinarily implies higher random- and multiple-coincidence background activity. However, the excellent energy resolution and tight energy windowing $(19,23)$, as well as the ability to estimate the direction of incoming photons (14), can help mitigate these potential problems.

\section{ADVANCED CAPABILITIES FOR PET}

With high-resolution 3D positioning and precise energy measurement of each of multiple interactions per annihilation photon event, these new detectors are capable of determining the interaction sequence (Fig. 2A) (13). If the first interaction in a multi-interaction sequence is resolved, the event is assigned to the correct line of response, resulting in the best possible spatial and contrast resolution performance. The interaction sequencing also enables an estimation of each incoming photon's direction (Fig. 2B). This information would yield new capabilities to PET such as an alternative method for rejecting random photon coincidences based on an incident photon direction window setting (Fig. 2C) (14). The incoming photon angle estimate also provides the ability to use rather than reject single (unpaired), multiple $(>2)$, and tissue-scattered photon coincidences during the image reconstruction process (Fig. 2C) $(5,14,24,25)$. For example, in this new paradigm, random and multiple photon coincidence events would be addressed on the basis of observing which photons are or are not colinear, and true coincident photon pairs can be extracted from a triple coincidence photon event that would otherwise be thrown out (14). If successful, these new capabilities to retain photon events that are normally rejected enable a new approach to substantially increase photon sensitivity (i.e., more counts collected in a given acquisition time) and, thus, significantly enhance image signal-to-noise ratio. The enhanced spatial and contrast resolution enabled through accurate sequencing of photon interactions in the detector is demonstrated in Figure 3.

\section{CONCLUSION}

We are exploring new 3D position-sensitive photon detection concepts for PET and incorporating them into both clinical and preclinical imaging systems under construction. These innovative detection concepts are capable of achieving submillimeter spatial resolution, without compromise to other performance parameters important to PET such as energy resolution, time resolution, photon sensitivity, and counting rate performance. In addition, the 3D positioning feature, together with novel event-processing algorithms, enable enhanced spatial resolution and contrast resolution and contrast recovery, as well advanced capabilities for PET such as the ability to retain a substantial fraction of events we normally reject, such as single photons, photon coincidences where at least one photon has scattered in tissue, and 


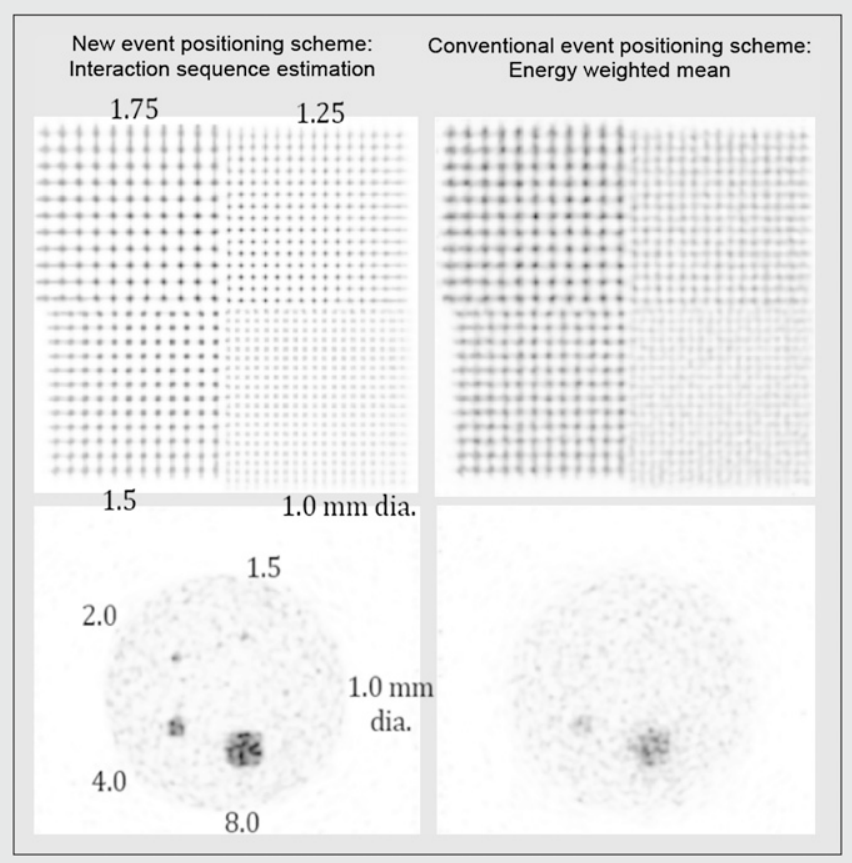

FIGURE 3. Image reconstruction results from simulated PET acquisition of sphere resolution phantom (top row: sphere spacing = twice sphere diameters listed) and rod contrast phantom (bottom row: outer rod diameter $=5$ $\mathrm{cm}$; 10:1 activity concentration ratio) in box-shaped PET system with $8 \times 8 \times$ $8 \mathrm{~cm}$ imaging field of view built with $1 \times 1 \times 5 \mathrm{~mm}$ resolution detection elements. Data were reconstructed with 100 sub-iterations of list-mode 3D ordered-subsets expectation maximization algorithm. In these data, coordinates of first interaction in multi-interaction photon events were estimated using new algorithm (left image) that determines most likely sequence of interactions (13), and energy-weighted mean (right image), which positions events at energy-weighted mean position of interactions, which is the only information available for conventional PET detector configuration (e.g., Fig. 1A). Accurately positioning event at first interaction in detector preserves best spatial resolution and contrast resolution. dia. $=$ diameter.

multiple ( $>2$ ) photon coincidences, to effectively boost photon sensitivity and image signal-to-noise ratio or make more efficient use of patient dose. If successful, these advances can be exploited to help visualize and quantify lower concentration of a PET probe for more sensitive study of subtle molecular signatures and pathways of disease in the clinic or in preclinical research.

\section{ACKNOWLEDGMENTS}

The developments described in this paper were funded in part by National Institute of Health grants R01 CA119056, R01 CA119056S1 (ARRA), R01CA120474, and R01EB011552 and Department of Energy grant DESC0005290. No other potential conflict of interest relevant to this article was reported.

\section{REFERENCES}

1. Levin CS, Hoffman EJ. Calculation of positron range and its effect on positron emission tomography system spatial resolution. Phys Med Biol. 1999;44:781-799.

2. Tai YC, Laforest R. Instrumentation aspects of animal PET. Annu Rev Biomed Eng. 2005; 7:255-285.

3. Lewellen TK. Recent developments in PET detector technology. Phys Med Biol. 2008;53:R287-R317.

4. Levin CS, Zaidi H. Current trends in preclinical PET system design. PET Clin. 2007;2:125-160.

5. Levin CS. New imaging technologies to enhance the molecular sensitivity of positron emission tomography. Proc IEEE. 2008;96:439-467.
6. Peng BH, Levin CS. Recent developments in PET instrumentation. Curr Pharm Biotechnol. 2010;11:555-571.

7. Levin CS. Instrumentation and methods to combine small animal PET with other imaging modalities. In: Weissleder R, Ross BD, Rehemtulla, Gambhir SS, eds. Molecular Imaging: Principles and Practice. Shelton, CT: People's Medical Publishing House-USA; 2010:99-117.

8. Phelps ME, Huang SC, Hoffman EJ, et al. An analysis of signal amplification using small detectors in positron emission tomography. J Comput Assist Tomogr. 1982;6:551-565.

9. Levin CS. Application-specific small field-of-view nuclear emission imagers in medicine. In: Aarsvold J, Wernick M, eds. Emission Tomography: The Fundamentals of PET and SPECT. San Diego, CA: Elsevier-Academic Press; 2004:293-334.

10. Levin CS, Tornai MP, Pani R, et al. Proceedings of the 2004 workshop on the nuclear radiology of breast cancer: Rome (Italy), October 22-23, 2004. Phys Med. 2006;21(suppl 1):1-140.

11. Cherry SR. Advances in PET imaging technology. J Nucl Med. 2006; $47: 1735-1745$.

12. Levin CS. Design of a high-resolution and high-sensitivity scintillation crystal array for PET with nearly complete light collection. IEEE Trans Nucl Sci. 2002;49-5(1):2236-2243.

13. Pratx G, Levin CS. Bayesian reconstruction of photon interaction sequences for high-resolution PET detectors. Phys Med Biol. 2009;54:5073-5094.

14. Chinn G, Levin CS. A maximum NEC criterion for Compton collimation to accurately identify true coincidences in PET. IEEE Trans Med Imaging. 2011;30:1341-1352.

15. Vandenbroucke A, Foudray AMK, Olcott PD, et al. Performance characterization of a new high resolution PET scintillation detector. Phys Med Biol. 2010;55:5895-5911.

16. Gu Y, Matteson JL, Skelton RT, et al. Study of a high resolution, 3-D positioning cross-strip cadmium zinc telluride detector for PET. Phys Med Biol. 2011;56:1563-1584.

17. Zhang J, Foudray AMK, Olcott PD, et al. Performance characterization of a novel thin position-sensitive avalanche photodiode for $1 \mathrm{~mm}$ resolution positron emission tomography. IEEE Trans Nucl Sci. 2007;54:415-421.

18. Spanoudaki VCh, Levin CS. Investigating the temporal resolution limits of scintillation detection: comparison between experiment and simulation. Phys Med Biol. 2011;56:735-756.

19. Habte F, Foudray AMK, Olcott PD, et al. Effects of system geometry and other physical factors on photon sensitivity of high resolution positron emission tomography. Phys Med Biol. 2007;52:3753-3772.

20. Lau FWY, Vandenbroucke A, Reynolds PD, et al. Analog signal multiplexing for PSAPD-based PET detectors: simulation and experimental validation. Phys Med Biol. 2010;55:7149-7174.

21. Gu Y, Pratx G, Lau FWY, et al. Effects of multiple-interaction photon events in a high resolution PET system that uses 3-D positioning detectors. Med Phys. 2010;37:5494-5508.

22. Reynolds PD, Olcott PD, Pratx G, et al. Convex optimization of coincidence time resolution for a high resolution PET system. IEEE Trans Med Imaging. 2011;30:391-400.

23. Levin CS, Habte F, Foudray AMK, et al. Impact of high energy resolution detectors on the performance of a PET system dedicated to breast cancer imaging. Phys Med. 2006;21 (suppl 1):28-34.

24. Chinn G, Foudray AMK, Levin CS. A method to include single photon events in image reconstruction for a $1 \mathrm{~mm}$ resolution PET system. IEEE Nuclear Science Symposium and Medical Imaging Conference Record. 2006:1740-1745.

25. Chinn G, Foudray AMK, Levin CS. Accurately positioning and incorporating large angle tissue scattered photons into PET image reconstruction. IEEE Nuclear Science Symposium and Medical Imaging Conference Record. 2006:1746-1761. 